\title{
Particle Swarm Optimization Compared to Other Heuristic Search Techniques for Pipe Sizing
}

\author{
C. R. Suribabu ${ }^{*}$ and T. R. Neelakantan \\ School of Civil Engineering, SASTRA Deemed University, Thanjavur 613 402, India
}

\begin{abstract}
An economical design of water distribution network is the aim of any agency dealing with water supply distribution. The fund needed for the construction, maintenance and operations of these systems requires an achievement of a good compromise between technical and economical aspects. Though the overall planning process of water distribution networks consists of three phases: layout, design and operation and each phase is not independent, but from a technical point of view, each can be formulated and solved as a separate problem. Several methods are available for designing a water distribution networks. In the present research, optimal design of water distribution network is experimented with particle swarm optimization (PSO) under dynamic adaptation and it is compared with genetic and simulated annealing algorithm. Particle swarm optimization is a relatively new generation of combinatorial meta-heuristic evolutionary algorithm that utilizes the swarm intelligence to achieve the goal of optimizing a specified objective function. This algorithm uses the cognition of the individuals and social behavior in the optimization process. The effectiveness of this algorithm is reported in terms of number of function evaluations and CPU time.
\end{abstract}

Keywords: Optimization, particle swarm optimization, swarm intelligence, water distribution networks

\section{Introduction}

A water distribution system is an essential infrastructure for supplying water to domestic as well as industrial uses. It connects consumers to sources of water, using hydraulic components, such as pipes, valves, pumps and tanks. The design of such a system is a multifarious task involving numerous interrelated factors requiring careful consideration in the design process. Important design parameters include water demand, minimum pressure requirements, topography, system reliability and economics of piping, pumping and energy uses. Two sets of simultaneous equations are needed to solve a network simulation problem. The first set of equations describes that conservation of flow must be satisfied at each network junction. The second set of equations is based on the principle of conservation of energy, which is described by the non-linear relation between flow and head loss in each pipe. Solving the simultaneous equations using iterative procedure requires the aid of computer as the equations are non-linear and number of equations is large. Hence, the developments in the field of computing place a great role in the planning and design of water distribution pipe network. Finding the optimal design of a distribution system is a challenging task as it is a time-consuming work involving reliability-cost trade-off. Selecting a network configuration with minimum pipe-cost and maximum reliability is a complex process. Numerous works have been reported in the literatures for the optimal design and optimal design considering certain reliability factors. Wide varieties of

\footnotetext{
* Corresponding author: suribabu@civil.sastra.edu
}

optimization tools have been applied in the past 30 years for optimal design of networks which include linear programming, different kinds of non-linear programming, heuristic methods like the genetic algorithm, simulated annealing technique, and colony algorithm, etc. Each method has its own advantages and disadvantages in formulation, speed of solving, handling nonlinearity, efficiency, etc. The last few decades have witnessed a growing interest in adapting mathematical models that can mimic natural biological processes into engineering design algorithms, thereby increasing the robustness and efficiency of these tools. Genetic algorithms (GA) are search and optimization tools, which work differently, compared to classical search and optimization methods. The primary reasons for their success are their broad applicability, ease of use and global perspective (Goldberg, 1989). The application of genetic algorithm on the optimal design of pipe networks are reported in Goldberg and Kuo (1987), Simpson et al. (1994), and Savic and Walters (1997). Goldberg and Kuo (1987) applied the genetic algorithms for optimizing operations of a gas pipeline. Simpson et al. (1994) were first applied genetic algorithm for optimal design of water distribution network. Unknown decision variables are coded as binary strings and three simple operators of GA are used in evaluation process. It is reported that GA helps to find near global optimal at the expenses of few evaluations compared to the size of the search space. Wu and Simpson (1996) used messy genetic algorithms for optimization of water distribution network through two different formulations, namely discrete pipe sizes model and split pipe cost model. A messy genetic algorithm uses variable-length strings, a threshold genetic selection and messy operators of cut and splice. Savic and Walter (1997) 
applied genetic algorithm to two well-known problems available in literature and it is reported that the result obtained is promising when compared with other methods. Halhal et al. (1997) experimented a structured messy genetic algorithm for optimal rehabilitation of a water distribution system. The structured messy genetic algorithm has some features of the messy GA such as coding scheme and variable length strings. It is based on progressively building up the complexity of the individuals in successive populations of solutions, in a structured manner, partially imitating the natural evolution of complex life forms from single cell organisms. Montesinos et al. (1999) proposed a modified GA for water distribution optimization by introducing several changes in the selection and mutation processes of a simple GA. Dandy and Engelhardt (2001) applied GA for optimal schedule for the replacement of the water pipes with the aim of minimizing the present value of capital repair and damage costs.

Cunha and Sousa (1999) used simulated annealing algorithm for optimal design of water distribution network. The simulated annealing procedure simulates the process of slow cooling of molten metal to achieve the minimum function value in a minimization problem. The cooling phenomenon is simulated by controlling a temperature-like parameter introduced with the concept of the Boltzmann probability distribution. Maier et al. (2003) applied ant colony algorithm in optimal design and rehabilitation of water distribution network. It is highlighted that the computational performance and searching of global solution is promising compared with the genetic algorithms. Liong and Atiquzzaman (2003) proposed shuffled complex algorithms for optimal designs. Eusuff and Lansey (2003) proposed a shuffled frog-leaping algorithm for water distribution network optimization, which mimics the culture of frogs and the algorithm is developed based on the concept of memetic evolution in the form of infection of ideas from one individual to another in a local search. A shuffling strategy allows for the exchange of information between local searches to move toward a global optimum. In present work, an attempt is made for applying Particle Swarm Optimization (PSO) with dynamic adaptation for optimal design of water distribution network.

The particle swarm optimization is a relatively new generation of combinatorial metaheuristic algorithms, which is based on a metaphor of social interaction, namely bird flocking or fish schooling. PSO utilizes both 'social' and 'cognition' components of individuals. Adjustment in the behavior of an individual according to the successful individual in the neighborhood forms the 'social' component while the adjustment due to individual's isolated experience forms the 'cognition' component. PSO has gained importance due to its comparative ease of operation and ability to quickly reach at an optimal or near optimal solution. PSO is considered as a potential competitor to other promising techniques like genetic algorithms, simulated annealing and tabu search. PSO has many resemblances with evolutionary computation techniques such as genetic algorithms (GA). However the information sharing mechanism in PSO is significantly different from others. In GAs, the chromosomes share information with each other and so the whole population involves in the next generation. In PSO, only the global best and local best gives information to others and so it is a one-way information sharing mechanism. The evolution only looks for the best solution. In this paper, an attempt is made to apply the PSO for the optimization of a water distribution system. The objectives of this study are to apply the PSO under dynamic adaptation for optimal design of water distribution network and to compare the performance of PSO and other algorithms for two benchmark water distribution system optimization case studies.

\section{Water Distribution Optimization Model}

In this study, the layout of the network, the nodal demand, and the minimum pressure requirements at different nodes are assumed known. The water distribution network design is formulated as a least-cost optimization problem with a selection of commercially available discrete pipe sizes as the decision variables. The optimization of given layout and demand at various nodal points of water distribution network is to find the combination of commercial pipe diameters that gives the least cost network by fulfilling the constraints on pressures. Minimization of the total cost of the distribution system may be expressed mathematically as

Minimize Cost $=f\left(D_{1}, D_{2}, \ldots . D_{N}\right)$

where $D_{l}$ represents pipe diameter for link 1 and $N$ is the total number of links in the network. The network cost is calculated as the sum of the pipe costs where pipe costs are expressed in terms of cost per unit length. Total network cost is computed as follows:

Cost $=\sum_{i=1}^{N} c\left(D_{i}\right) \cdot L_{i}$

where $c\left(D_{i}\right)$ is the cost per unit length of the $i^{\text {th }}$ link with diameter $D_{i}$, and $L_{i}$ is the length of $i^{\text {th }}$ link. For a given layout, lengths are fixed and so diameters are the decision variables. This objective function needs to be minimized subject to a set of constraints as follows.

Continuity of flow in each node should be maintained in the network. The quantity of flow entering the node should be equal to the quantity of flow leaving the node. The quantity of flow leaving the node includes the external demand and flow going out through other pipes emerging from node. This is expressed in the mathematical form as

$\sum_{i \in \text { in }, n} Q_{i}=\sum_{j \in \text { out }, n} Q_{j}+N D_{n} \quad \forall n \in N N$

where $Q=$ pipe flow; $N D_{n}=$ Demand at node n; in, $n=$ set of pipes entering to the node $\mathrm{n}$; out, $n=$ set of pipes emerging from node $\mathrm{n}$; and $N N=$ node set. 
The total head loss around the closed path (loop) should be equal to zero or the head loss along a path between nodes should be equal to the difference in elevation.

$\sum_{i \in \text { loop } p} h f_{i}=\Delta H \quad \forall p \in N L$

where $h f_{\mathrm{i}}=$ head loss due to friction in pipe i; $N L=$ loop set; $\Delta H=$ Difference between nodal heads at both ends, $\Delta H=0$, if the path is closed.

The Hazen-Williams head loss equation for pipe $i$ of connecting nodes $j$ and $k$ is given as follows:

$H_{j}-H_{k}=h f_{i}=\frac{\alpha L_{i} Q_{i}\left|Q_{i}\right|^{0.852}}{C_{H W, i}{ }^{1.852} D_{i}^{4.87}} \quad \forall j \in N P$

where $N P=$ number of pipes; $C_{H W}=$ Hazen-Williams coefficient; $D_{i}=$ Diameter of the pipe $i ; L_{i}=$ length of the pipe $i$; $\alpha=$ Conversion factor which depend on the units. Different values of $\alpha$ are found in literature - as low as 10.4516 to as high as 10.9031 (Savic and Walters, 1997).

The pressure head in all nodes should be greater than the prescribed minimum pressure head as follows:

$H_{n} \geq H_{\min }$

where $H_{n}=$ Pressure head at node, $\mathrm{n} ; H_{\min }=$ Minimum required pressure head. The diameter of the pipes should be within available commercial size:

$D_{j}=[D], \quad \forall i \in N P$

\section{Particle Swarm Optimization}

The particle swarm optimization (PSO) was originally designed by Kennedy and Eberhart (1995) to model naturally occurring swarming behaviors in a computer program, and called their creation as particle swarm optimization. A particle represents a bird or a bee or a fish, or any other type of natural agent that exhibits a swarming behavior. Such a technique involves simulating social behavior among individuals (particles) 'flying' through a multidimensional search space, each particle representing a single interaction of all search dimensions. The particle evaluate their positions relative to a goal (fitness) at every iteration, and particles in a local neighborhood share memories of their 'best' positions, then use those memories to adjust their own velocities, and thus subsequent positions. As a consequence, the particles move towards the global best solution. The original PSO formula developed by Kennedy and Eberhart (1995) was greatly improved by Shi and Eberhert (1998) with the introduction of an inertia parameter $\omega$ that increases the overall performance of PSO. The inertia weight is employed to control the influence of the previous history of velocities on the current velocity, thus to influence the trade-off between global (widespread) and local (narrow) exploration abilities of the 'flying points'. A larger inertia weight facilitates global exploration (searching new areas) while a smaller inertia weight tends to facilitate local exploration to fine-tune current search area. Suitable selection of the inertia weight can provide a balance between the global and local exploration abilities and thus reduces the number of iterations required to reach optimum. Thus the inertia weight helps generate non-dominated solutions and maintains diversity.

The initial velocity matrix can be set as follows:

$v_{m, n}=x_{\min , n}+r_{1} *\left[x_{\max , n}-x_{\min , n}\right]$

where $m$ is the population size, $n$ is the number of decision variables, $x_{\min , n}$ is the minimum value of individual in population and $x_{\max , n}$ is the maximum value of the individual in population. Thus the new positions of particles can be computed using the following equation:

$x_{m, n}(t+1)=x_{m, n}(t)+v_{m, n}(t+1)$ lows:

The velocity matrix is updated at each iteration as fol-

$v_{m, n}(t+1)=v_{m, n}(t) * w+C_{1} * r_{1} *\left(p_{m, n}-x_{m, n}\right)+C_{2} * r_{2} *\left(g_{n}-x_{m, n}\right)$

where $p_{m, n}$ is the local best ( a particle's best so far), $g_{n}$ is the global best (best position so far among all particles), $w$ is a non-linear inertia weight, taken as $\left(n / 3+r_{3} / 2\right)^{n}, C_{1}$ and $C_{2}$ are two positive constants, and $r_{1}, r_{2}$ and $r_{3}$ are random numbers between 0 and 1 .

The search can be terminated if one of the following criteria is satisfied: (a) the number of iterations since the last change of the best solution is greater than a pre-specified number or (b) the number of iterations reaches the maximum allowable number. The flow chart for PSO is presented in Figure 1.

PSO has many resemblances with evolutionary computation techniques such as genetic algorithms (GAs). The system is initialized with a population of random solutions and searches for optima through updating. However, unlike GA, PSO does not have any evolution operators such as crossover and mutation. Compared to GA, the advantages of PSO are that PSO is easy to implement and there are few parameters to be adjusted. Though GA is a useful algorithm for optimization, drawbacks of premature convergence and weak exploitation capabilities, especially in optimizing continuous multi-model 
functions, are well known (Goldberg, 1989; Chelouah and Siarry, 2000, 2003; Wang and $\mathrm{Wu}, 2004)$. Loss of diversity in the population often causes premature convergence (provides a local optimal solution). Optimization problems involving many local optima suffer from excessively slow convergence when solved with GA. This slow convergence is because of the fact that standard genetic algorithm does not exploit the neighborhood information. Such a slow convergence problem has been highlighted by a few authors (Forrest and Mitchell, 1993; Schenecke and Vornberger, 1997; Ching-Hung et al., 1998; Olivier, 1998; Wang and Wu, 2004). Further, genetic algorithm converges fast initially and slows down to a relatively inefficient rate after a number of iterations. When a variable is discrete and expressed in binary form, moving of decision variable to neighborhood point is rare through crossover and mutation process. Even good solutions are made in multiple copies through the selection process in each generation; when these solutions undergo crossover and mutation process, more number of poor solutions are generally resulted.

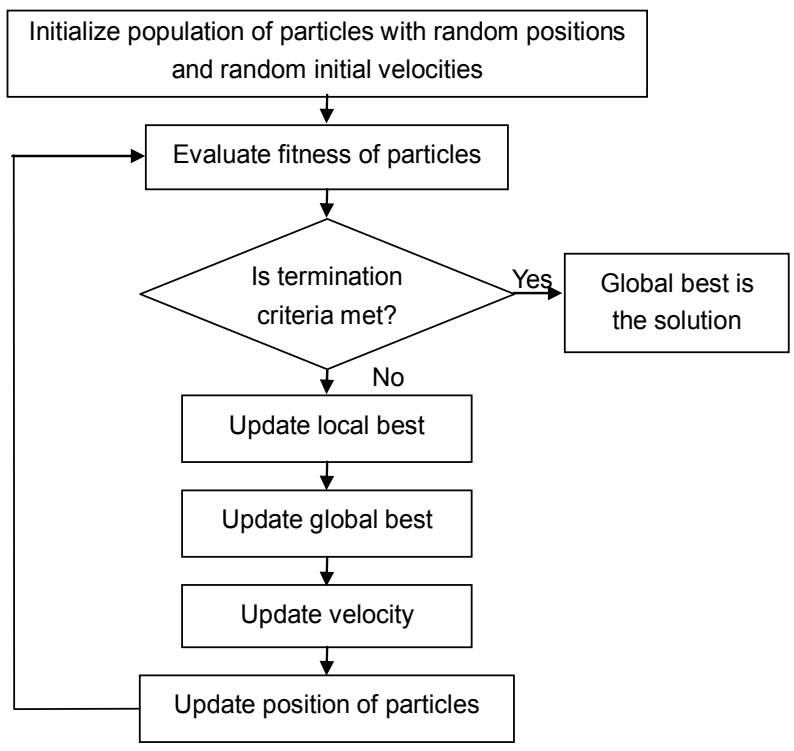

Figure 1. Flow chart describing the PSO algorithm.

\section{Optiwaternet}

A combined simulation-optimization model is applied in this study. The optimization model is the outer driver model and simulation is the inner model. The United States Environmental Protection Agency (USEPA) developed freeware software called EPANET (Rossman, 2000) for the simulation of water distribution networks. They also provide EPANET Programmer's Toolkit that is a dynamic link library (DLL) of functions, which allows developers to customize EPANET's computational engine for the user's specific needs. In the present research, Toolkit DLL file is included as a module and its library functions are used in Visual Basic code for combined optimization and simulation model.
The steps involved in the development of OPTIWATERNET are presented in Figure 2. The algorithms used for optimizing the water distribution networks are genetic algorithm (GA), simulated annealing algorithm (SA), non-equilibrium simulated annealing algorithm (NESA) and particle swarm optimization with non-linear inertia weight. In order to compare the computational time needed for finding the optimal solution for example networks, a separate module is developed for GA, SA and NESA similar to PSO and interfaced with EPANET's computation engine. The detailed description of GA, SA and NESA modules are not presented here since the focus of the present work is to explain the application PSO with dynamic adaptation. Optimization techniques used in this study is experimented with equal number of trial runs in order to compare the number of function evaluations and computational time to obtain the same cost solution.

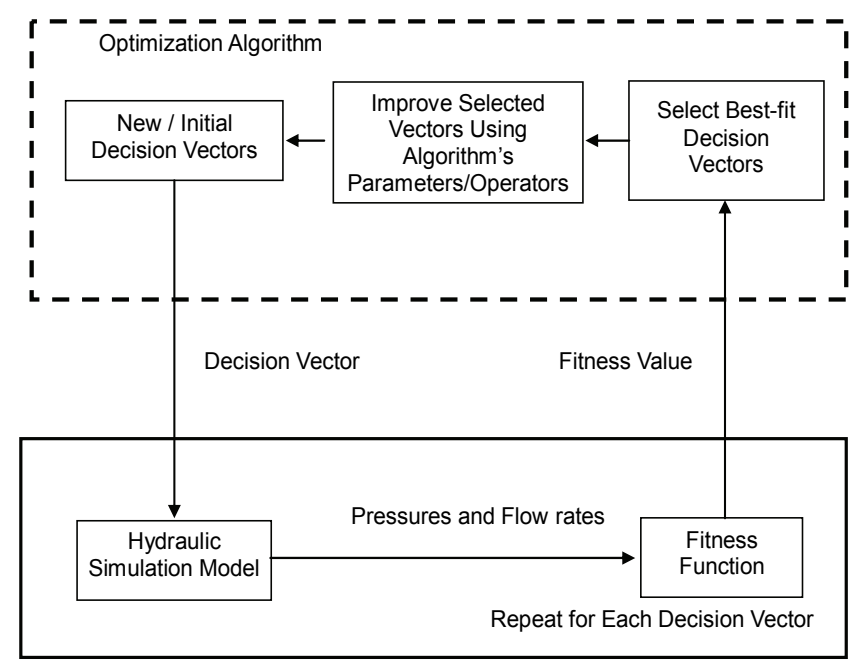

Figure 2. Flow chart of OPTIWATERNET.

Table 1. Node Details of Two-loop Network

\begin{tabular}{llll}
\hline Node & Elevation $(\mathrm{m})$ & $\begin{array}{l}\text { Min. Pressure } \\
(\mathrm{m})\end{array}$ & $\begin{array}{l}\text { Demand } \\
\left(\mathrm{m}^{3} / \mathrm{hr}\right)\end{array}$ \\
\hline 1 & 210 & - & -1120 \\
2 & 150 & 30 & 100 \\
3 & 160 & 30 & 100 \\
4 & 155 & 30 & 120 \\
5 & 150 & 30 & 270 \\
6 & 165 & 30 & 330 \\
7 & 160 & 30 & 200 \\
\hline
\end{tabular}

\section{Design Examples}

\subsection{Example 1: Two-Loop Network}

The water distribution network shown in Figure 3 is used for demonstration. This network was first used by Alperovits 
and Shamir (1977) optimization studies. It is a simple smallsize benchmark network commonly used for testing the optimization algorithms in water distribution network studies. The network consists of 8 links, 6 demand nodes, and 1 reservoir (node 1). The node details of this network are given in Table 1. The minimum required pressure at all demand nodes is $30 \mathrm{~m}$. Each link is $1000 \mathrm{~m}$ long. Alperovits and Shamir (1977) and subsequently many others used a constant Hazen-Williams coefficient of 130 for all pipes. Table 2 shows the costs of the pipes of various diameters.

Table 2. Cost Data of Pipes for Two-loop Network

\begin{tabular}{lll}
\hline Diameter (in) & Diameter $(\mathrm{mm})$ & Unit Cost (unit $/ \mathrm{m})$ \\
\hline 1 & 25.4 & 2 \\
2 & 50.8 & 5 \\
3 & 76.2 & 8 \\
4 & 101.6 & 11 \\
6 & 152.4 & 16 \\
8 & 203.2 & 23 \\
10 & 254.0 & 32 \\
12 & 304.8 & 50 \\
14 & 355.6 & 60 \\
16 & 406.4 & 90 \\
18 & 457.2 & 130 \\
20 & 508.0 & 170 \\
22 & 558.8 & 300 \\
24 & 609.6 & 550 \\
\hline
\end{tabular}

In optimization models, continuous diameters and split pipes (two or more diameter/size pipes form a link) were prominently used a decade ago when suitable algorithms were not available for using discrete commercial diameters/sizes. However, the use of commercial sizes is preferred as they represent realities more. Since two-loop network possess eight pipes and fourteen discrete pipe diameter sets for each pipe, it ultimately forms the search space containing $14^{8}=1.48 \times 10^{9}$ different possible designs. The number of the explicit decision variables is eight and other decision variables, like demand and pressure at various nodes will be implicitly handled by hydraulic network solver. Table 3 shows solutions obtained by different authors using different techniques and also methods considered in the present study for the two-loop network. The least cost obtained using discrete diameters is 419,000 units with the $457.2,254,406.4,101.4,406.4,101.4,406.4,254$, 254 and $25.4 \mathrm{~mm}$ pipes respectively for links 1 through 8 . Figure 4 presents the evolution of optimal solution for two-loop network. The solution obtained by Savic and Walter (1997), Cunha and Sousa (1999), Eusuff and Lansey (2003) and Liong and Atiquzzaman (2004) have exactly the same least cost solution. It can be seen from Table 3 that PSO has taken only two seconds of CPU time while running the module using PC IV $65 \mathrm{MHz}$. Even though there is a decreasing trend of computation cost, it is essential to explore the fast converging algorithms, which will be more appropriate in handling large size problem and handling several constraints. In the present study, few trial runs are performed with different permutation and combinations of $C_{1}, C_{2}$ and $n$ value of inertia weight $(n / 3$ $+r n d / 2)^{n}$.

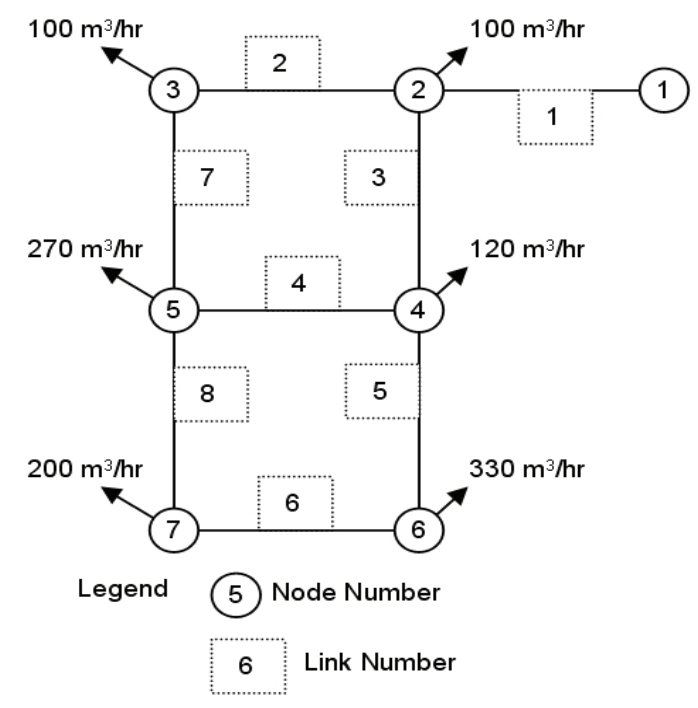

Figure 3. Schematic diagram of the two-loop network.

From above analysis, it is indicated that the least cost for two-loop network (419,000 units) was obtained with minimum number of function evaluations at $C_{1}$ and $C_{2}$ with 2 and

Table 3. Solutions for Two-Loop Network

\begin{tabular}{|c|c|c|c|c|c|}
\hline Sl. No & Authors & Technique used & $\begin{array}{l}\text { Optimal cost } \\
\text { (Units) }\end{array}$ & $\begin{array}{l}\text { Number of } \\
\text { function evaluation }\end{array}$ & CPU time (s) \\
\hline 1. & Savic and Walter (1997) & Genetic Algorithm & 419,000 & 65,000 & 600 \\
\hline 2. & Cunha and Sousa (1999) & Simulated Annealing Algorithm & 419,000 & 25,000 & 40 \\
\hline 3 & Eusuff and Lansey (2003) & Shuffled leap frog algorithm & 419,000 & 11,155 & Not available \\
\hline 4 & Liong and Atiquzzaman (2004) & Shuffled complex algorithm & 419,000 & 1,019 & 18 \\
\hline 5 & Present work & Particle swarm optimization & 419,000 & 760 & 2 \\
\hline 6 & Present work & Genetic Algorithm & 420,000 & 58,380 & 86 \\
\hline 7 & Present work & Simulated Annealing & 419,000 & $2,68,200$ & 43 \\
\hline 8 & Present work & Non-Equilibrium Simulated Annealing & 419,000 & $1,09,957$ & 25 \\
\hline
\end{tabular}


1.5 for $n$. From the trial runs, it is identified that the value of inertia weight in PSO plays a vital role in reaching the optimal solution. The population size, the random numbers and values of $C_{1}$ and $C_{2}$ and the inertia weight all seems can affect the optimization process. The analysis was also repeated with a constant inertia of 1 in PSO algorithm instead of dynamic inertia weight. However, this does not provide a better result. In this case, out of ten trials, the mean number of evaluation required to reach the optimal solution is 212,175 . This clearly shows that the value of inertia weight in PSO plays a vital role in reaching an optimal solution. However, with only limited attempts being made, it is seldom to deduce some specific values or ranges for these parameters. The results obtained by GA for the two-loop problem is slightly higher than that of PSO. Comparing the results of PSO and GA, it is evident that the PSO is performing definitely better than GA. With GA, even after 58,380 function evaluations, an optimum cost of 419,000 units could not be achieved for the different cases tried, where as the PSO could reach the optimum at the expanse of 760 function evaluations in 2 seconds. Number of function evaluations reported by the simulated annealing proposed by Cunha and Sousa (1999) and the present work hold opposing view because of considered initial configuration as an initialization to the algorithm and simulation model used for analyzing the network. Even though number of function evaluations taken for obtaining same solution is different from that of Cunha and Sousa (1999), the CPU time for both cases are more or less the same. Cardoso et al. (1994) proposed a non-equilibrium simulated annealing algorithm (NESA), in which the temperature will be decreased as soon as better solution is found while trying to reach equilibrium through large transitions at any fixed temperatures. Since the reduction of temperature is carried out before its reaching the thermal equilibrium, the method is named as Non-Equilibrium Simulated Annealing (NESA) algorithm. While optimizing the network through NESA, it is found that convergence to optimum is quite improved over simulated annealing. However, it has taken more number of function evaluations and CPU time than PSO. Though these independent results cannot be compared directly as randomness place a vital role in reaching the optimum, generally the number of function evaluations and CPU time are significantly less in the case of PSO.

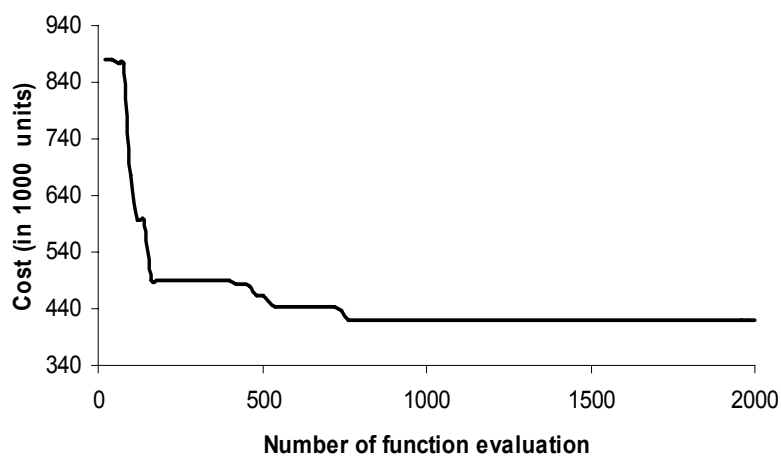

Figure 4. Evolution of optimal solution for the two-loop network design.

\subsection{Example 2: Hanoi Network}

The second test network (Figure 5) is a three-loop Hanoi city water distribution network, which consists of thirty-two nodes, thirty-four links and a reservoir. The input data for this problem is given in Fujiwara and Khang $(1990,1991)$ and is presented in Tables 4 and 5. This three-loop network is used by several researchers earlier (Sonak and Bhave, 1993; Eiger et al., 1994; Verma et al., 1997; Sherali et al., 1998; Cunha and Sousa, 1999; Eusuff and Lansey, 2003).

Table 4. Node and Link Data for Hanoi Network

\begin{tabular}{|c|c|c|c|c|}
\hline Node & $\begin{array}{l}\text { Demand } \\
\left(\mathrm{m}^{3} / \mathrm{hr}\right)\end{array}$ & Link & $\begin{array}{l}\text { Connecting } \\
\text { nodes }\end{array}$ & $\begin{array}{l}\text { Length } \\
\text { (m) }\end{array}$ \\
\hline 1 (Source) & $-19,940$ & 1 & $(1,2)$ & 100 \\
\hline 2 & 890 & 2 & $(2,3)$ & 1,350 \\
\hline 3 & 850 & 3 & $(3,4)$ & 900 \\
\hline 4 & 130 & 4 & $(4,5)$ & 1,150 \\
\hline 5 & 725 & 5 & $(5,6)$ & 1,450 \\
\hline 6 & 1,005 & 6 & $(6,7)$ & 450 \\
\hline 7 & 1,350 & 7 & $(7,8)$ & 850 \\
\hline 8 & 550 & 8 & $(8,9)$ & 850 \\
\hline 9 & 525 & 9 & $(9,10)$ & 800 \\
\hline 10 & 525 & 10 & $(10,11)$ & 950 \\
\hline 11 & 500 & 11 & $(11,12)$ & 1,200 \\
\hline 12 & 560 & 12 & $(12,13)$ & 3,500 \\
\hline 13 & 940 & 13 & $(10,14)$ & 800 \\
\hline 14 & 615 & 14 & $(14,15)$ & 500 \\
\hline 15 & 280 & 15 & $(15,16)$ & 550 \\
\hline 16 & 310 & 16 & $(16,17)$ & 2,730 \\
\hline 17 & 865 & 17 & $(17,18)$ & 1,750 \\
\hline 18 & 1,345 & 18 & $(18,19)$ & 800 \\
\hline 19 & 60 & 19 & $(19,3)$ & 400 \\
\hline 20 & 1,275 & 20 & $(3,20)$ & 2,200 \\
\hline 21 & 930 & 21 & $(20,21)$ & 1,500 \\
\hline 22 & 485 & 22 & $(21,22)$ & 500 \\
\hline 23 & 1,045 & 23 & $(20,23)$ & 2,650 \\
\hline 24 & 820 & 24 & $(23,24)$ & 1,230 \\
\hline 25 & 170 & 25 & $(24,25)$ & 1,300 \\
\hline 26 & 900 & 26 & $(25,26)$ & 850 \\
\hline 27 & 370 & 27 & $(26,27)$ & 300 \\
\hline 28 & 290 & 28 & $(27,16)$ & 750 \\
\hline 29 & 360 & 29 & $(23,28)$ & 1,500 \\
\hline 30 & 360 & 30 & $(28,29)$ & 2,000 \\
\hline 31 & 105 & 31 & $(29,30)$ & 1,600 \\
\hline \multirow[t]{3}{*}{32} & 805 & 32 & $(30,31)$ & 150 \\
\hline & & 33 & $(31,32)$ & 860 \\
\hline & & 34 & $(32,25)$ & 950 \\
\hline
\end{tabular}

The design of this network is restricted to select the six different commercial diameter pipes assumed available in the market. The solution space consists of $6^{34}$ numbers of solu- 
tions (i.e., $2.87 \times 10^{26}$ ) as there are 6 possible pipe diameters for each pipe. The number of explicit decision variables is 34 and other decision variables such as demand and pressure at various nodes will be implicitly handled by hydraulic network solver. For the head loss $\left(K Q^{I .85}\right)$, length $(L)$, diameter $(D)$ in meters and flow rate $(Q)$ in cubic meters per second, different values of $\alpha$ (Equation 5) are found in literatures ranging between 10.4516 to 10.9031 . In present study, the default value used in EPANET (i.e., 10.679) is applied. In the PSO analysis, few trial runs are performed with different permutation and combinations of $C_{1}, C_{2}$ and $n$ value of inertia weight similar that of first example. From the analysis, it is found that the least cost solution for Hanoi network $(6,081,087$ units) was obtained with minimum number of function evaluation at $C_{1}$ and $C_{2}$ with 2 and 1.5 for $n$. Table 6 gives the optimal solution of Hanoi network. Further, keeping the total number of the function evaluation as 25,000 , few trials are carried out by changing the $n$ values and it is found that at higher values of $n$ (greater than 2), convergence to global optimal slows down. However, it is very difficult to conclude about the appropriate value of $C_{1}, C_{2}$ and $n$ unless if it is tried with other kind of problems.

Table 5. Cost Data for Pipes for Hanoi Network

\begin{tabular}{lll}
\hline Diameter (in) & Diameter $(\mathrm{mm})$ & Unit Cost (unit $/ \mathrm{m})$ \\
\hline 12 & 304.8 & 45.73 \\
16 & 406.4 & 70.4 \\
20 & 508 & 98.38 \\
24 & 609.6 & 129.3 \\
30 & 762 & 180.8 \\
40 & 1,016 & 278.3 \\
\hline
\end{tabular}

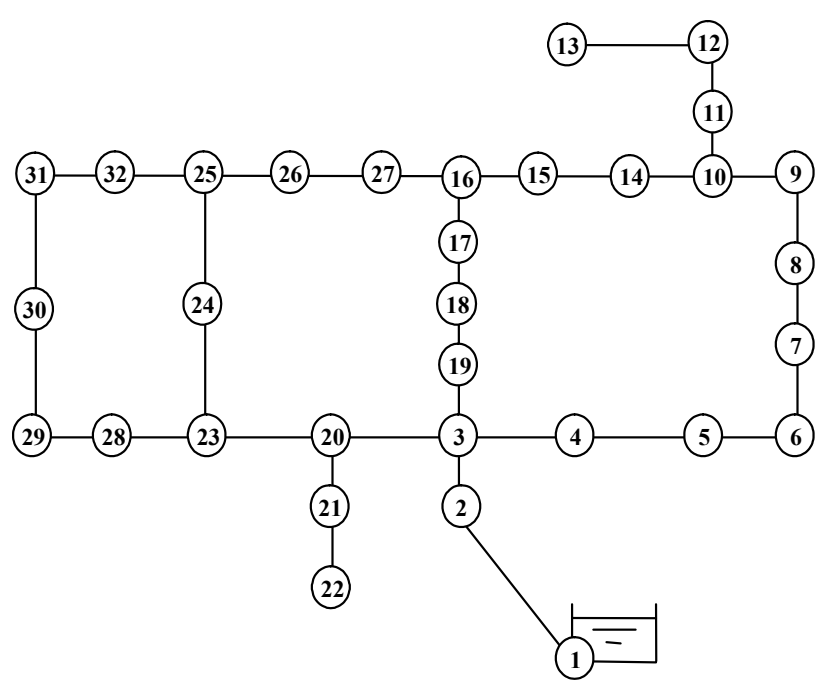

Figure 5. Layout Hanoi water distribution networks.

From Table 7, it is evident that the use of non-linear inertia weight in PSO reduces not only number of function evaluations but also the CPU time. It can be seen from Table 7 that the cost of solution obtained by the present method is marginally higher that of Savic and Walter (1997), Cunha and Sousa (1999) and Eusuff and Lansey (2003). However the solution of Cunha and Sousa (1999) and Savic and Walter (1997) violates pressure at 6 nodes and 2 nodes respectively. The solution presented by Cunha and Sousa (1999) has least cost of 6,056,000 units. However, this result has been obtained by a slightly different coefficient of Hazen-Willams equation ( $\alpha=10.5088$ ). While solved by EPANET, it is found that the pressure at the nodes 13,16, 17, 27, 29 and 30 falls between 29 and $30 \mathrm{~m}$. Savic and Walters (1997) reported a minimum cost of $6,073,000$ units by genetic algorithm. The pressure head at two nodes namely 13 and 30 falls between 29 and $30 \mathrm{~m}$. Hence these two solutions can be considered as infeasible solution in the present context. It can be seen from the Table 7 that the computation time for all other algorithms including results of other researchers is higher than PSO. As most of the earlier works are not employed EPANET's computational Engine for simulation, it is not worthwhile to compare its computation time with present works.

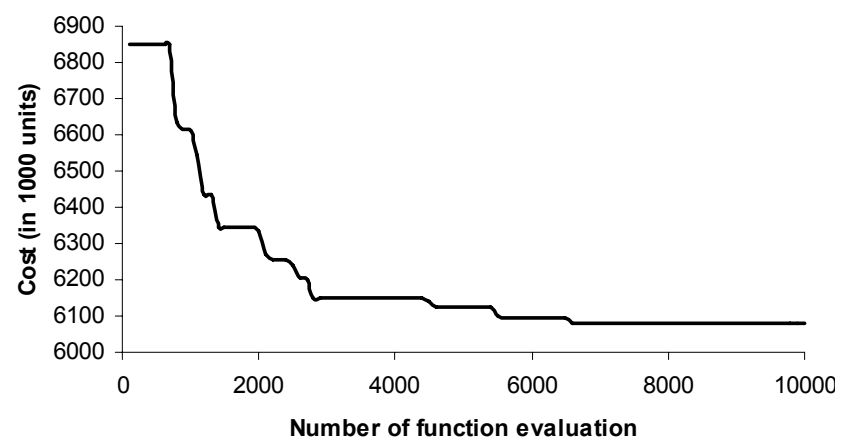

Figure 6. Evolution of optimal solution for the Hanoi network design.

\section{Conclusions}

Use of traditional optimization methods for solving pipesizing problems was limited due to difficulties in handling commercial pipe sizes. Introduction of meta-heuristic population based algorithms is more promising to handle such kind of problems. In this paper, the efficacy of dynamic non-linear inertia weight based particle swarm optimization was tested through solving the benchmark problems and compared with genetic and simulated annealing algorithms. The results of an application shows that PSO will be more promising technique for optimal design of water distribution network as the computation time and number of times the function need to be evaluated are less while comparing with the genetic algorithm and the simulated annealing algorithms. As the randomness plays a vital role, like GA and SA, it is seldom to conclude the optimal values of PSO parameters. However the introduction of non-linear inertia weight accelerates the exploration of a global solution. 
Table 6. Optimal Diameters (mm) for Hanoi Water Distribution Network

\begin{tabular}{lllllllllll}
\hline Link & 1 & 2 & 3 & 4 & 5 & 6 & 7 & 8 & 9 & 10 \\
\hline Diameter & 1016 & 1016 & 1016 & 1016 & 1016 & 1016 & 1016 & 1016 & 1016 & 762 \\
Link & 11 & 12 & 13 & 14 & 15 & 16 & 17 & 18 & 19 & 20 \\
Diameter & 609.6 & 609.6 & 508 & 406.4 & 304.8 & 304.8 & 406.4 & 609.6 & 508 & 1016 \\
Link & 21 & 22 & 23 & 24 & 25 & 26 & 27 & 28 & 29 & 30 \\
Diameter & 508 & 304.8 & 1016 & 762 & 762 & 508 & 304.8 & 304.8 & 406.4 & 304.8 \\
Link & 31 & 32 & 33 & 34 & & & & & & \\
Diameter & 304.8 & 406.4 & 406.4 & 609.6 & & & & & \\
\hline
\end{tabular}

Table 7. Solutions for Hanoi Water Distribution Network

\begin{tabular}{|c|c|c|c|c|c|c|}
\hline Sl. No & Authors & Technique used & $\begin{array}{l}\text { Number of function } \\
\text { evaluation }\end{array}$ & Cost (units) & Remark & $\begin{array}{l}\text { CPU time } \\
\text { (s) }\end{array}$ \\
\hline 1 & $\begin{array}{l}\text { Cunha and Sousa } \\
\text { (1999) }\end{array}$ & $\begin{array}{l}\text { Simulated Annealing } \\
\text { Algorithm }\end{array}$ & 53,000 & $6,056,000$ & $\begin{array}{l}\text { Pressure constraints } \\
\text { violated in } 6 \text { nodes }\end{array}$ & 7,200 \\
\hline 2 & $\begin{array}{l}\text { Savic and Walters } \\
\text { (1997) }\end{array}$ & Genetic Algorithm & $\begin{array}{l}\text { Not Available } \\
\text { In paper }\end{array}$ & $6,073,000$ & $\begin{array}{l}\text { Pressure constraints } \\
\text { violated in } 2 \text { nodes }\end{array}$ & 10,800 \\
\hline 3 & $\begin{array}{l}\text { Eusuff and Lansey } \\
\text { (2003) }\end{array}$ & $\begin{array}{l}\text { Shuffled leap frog } \\
\text { algorithm }\end{array}$ & 26,987 & $6,073,000$ & $\begin{array}{l}\text { Pressure constraints } \\
\text { violated in } 2 \text { nodes }\end{array}$ & $\begin{array}{l}\text { Not } \\
\text { available }\end{array}$ \\
\hline 4 & $\begin{array}{l}\text { Liong and } \\
\text { Atiquzzaman (2004) }\end{array}$ & $\begin{array}{l}\text { Shuffled complex } \\
\text { algorithm }\end{array}$ & 25,402 & $6,220,000$ & - & 660 \\
\hline 5 & Present work & $\begin{array}{l}\text { Particle Swarm } \\
\text { optimization }\end{array}$ & 6,600 & $6,081,087$ & - & 9 \\
\hline 6 & Present work & Simulated Annealing & $1,76,700$ & $6,081,087$ & & 160 \\
\hline 7 & Present work & Genetic Algorithm & $12,34,340$ & $6,081,087$ & & 1,800 \\
\hline 8 & Present work & $\begin{array}{l}\text { Non-Equilibrium } \\
\text { Simulated Annealing }\end{array}$ & 18,752 & $6,081,087$ & & 47 \\
\hline
\end{tabular}

\section{References}

Alperovits, E. and Shamir, U. (1977). Design of optimal water distribution systems. Water Resour. Res., 13(6), 885-900.

Cardoso, M.F., Salcedo, R.L. and de Azevedo, S.F. (1994). Non-equilibrium Simulated Annealing Algorithm: a faster approach to combinatorial minimization. Ind. Eng. Res., 33(8), 1908-1918.

Chelouah, R. and Siarry, P. (2000). A continuous genetic algorithm designed for the global optimization. J. Heuristics, 6, 191-213.

Chelouah, R. and Siarry, P. (2003). Genetic Nelder-Mead algorithms hybridized for a more accurate global optimization of continuous multi-minima functions. Eur. J. Oper. Res., 148, 335- 348

Ching-Hung, W., Tzung-pei, H. and Shian-shyong, T. (1998). Integrating fuzzy knowledge by genetic algorithms. IEEE Trans. Evol. Comput., 2(4), 138-149.

Cunha, M. and Sousa, J. (1999). Water distribution network design optimization: Simulated annealing approach. J. Water Resour. Plan. Manage., ASCE, 125(4), 215-221.

Dandy, G.C. and Engelhardt, M. (2001). Optimal scheduling of water pipe replacement using genetic algorithms. J. Water Resour. Plan. Manage., ASCE, 127(4), 214-223.

Eiger, G., Shamir, U. and Be-Tal, A. (1994). Optimal design of water distribution networks. Water Resour. Res., 30(9), 2637-2646.

Eusuff, M.M. and Lansey, K.E (2003). Optimization of water distribution network design using the shuffled frog-leaping algorithm. J. Water Resour. Plan. Manage., ASCE, 129(3), 210- 225.

Forrest, S. and Mitchell, M. (1993). What makes a problem hard for a genetic algorithm? Some anomalous results and their explanation. Mach. Learning, 13(2/3), 285-319.

Fujiwara, O. and Khang, D.B. (1990). A two-phase decomposition method for optimal design of looped water distribution networks. Water Resour. Res., 26(4), 539-549.

Fujiwara, O. and Khang, D.B. (1991). Correction to a two-phase decomposition method for optimal design of looped water distribution networks. Water Resour. Res., 27(5), 985-986.

Goldberg, D.E. (1989). Genetic Algorithms in Search, Optimization, and Machine Learning, Addison-Wesley, Mass.

Goldberg, D.E. and Kuo, C.H. (1987). Genetic Algorithms in pipeline optimization. J. Comput. Civil Eng., 1(2), 128-141.

Halhal, D., Walters, G.A., Ouazar, D. and Savic, D.A. (1997). Water network rehabilitation with structured messy genetic algorithm. J. Water Resour. Plan. Manage., ASCE, 123(3), 137-146.

Kennedy, J. and Eberhart, R.C. (1995). Particle swarm optimization, in Proc. of IEEE International Conference on Neural Networks, Piscataway, pp. 1942-1948.

Liong, S.Y. and Atiquzzaman, M. (2004). Optimal design of water distribution network using shuffled complex evolution. J. Inst. Eng., 44(1), 93-107.

Maier, H.R., Simpson, A.R., Zecchin, A.C., Foong, W.K., Phang, K.Y., Seah, H.Y. and Tan, C.L. (2003). Ant colony optimization for design of water distribution systems. J. Water Resour. Plan. Manage., ASCE, 129(3), 200-209.

Montesinos, P., Guzman, A.G. and Ayuso, J.L (1999). Water distribution network optimization using a modified genetic algorithm. 
Water Resour. Res., 35(11), 3467-3473.

Oliver, F. (1998). An evolutionary strategy for global minimization and its markov chain analysis. IEEE Trans. Evol. Comput., 2(3), 77-90.

Rossman, L.A. (2000). EPANET 2-User Manual, National Risk Management Research Laboratory, Office of Research and Development, US Environmental Protection Agency, Cincinnati, Ohio, USA.

Savic, D.A. and Walters, G.A (1997). Genetic algorithm for least cost design of water distribution networks. J. Water Resour. Plan. Manage., ASCE, 123(2), 66-77.

Schenecke, V. and Vornberger, O. (1997). Hybrid genetic algorithms for constrained placement problem. IEEE Trans. Evol. Comput., 1(4), 266-277.

Sherali, H.D., Totlani, R. and Loganathan, G.V. (1998). Enhanced lower bounds for the global optimization of water distribution networks. Water Resour. Res., 34(7), 1831-1841.

Shi, Y. and Eberhart, R.C. (1998). Particle swarm optimizer, in Proc. of the IEEE International Conference on Evolutionary Computation, pp. 69-73.

Simpson, A.R., Dandy, G.C. and Murphy, L.J (1994). Genetic algorithm compared to other techniques for pipe optimization. $J$. Water Resour. Plan. Manage., ASCE, 120(4), 423-443.

Sonak, V.V. and Bhave, P.R. (1993). Global optimum tree solution for single-source looped water distribution networks subjected to a single loading pattern. Water Resour. Res., 29(7), 2437- 2443.

Varma, K.V.K., Narasimhan, S. and Murthy, B.S. (1997). Optimal design of water distribution systems using an NLP method. J. Environ. Eng., ASCE, 123(4), 381-388.

Wang, H.F. and Wu, K.Y. (2004). Hybrid genetic algorithm for optimization problems with permutation property. Comput. Oper. Res., 31(14), 2453-2471.

Wu, Z.Y. and Simpson, A.R. (1996). Messy Genetic Algorithms for Optimization of Water Distribution Systems, Research Report No. R140, Department of civil and environmental engineering, The University of Adelaide, Australia, pp. 1-60. 